EDITORA

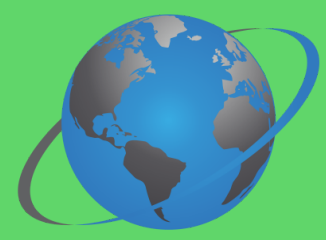

OMNIS SCIENTIA

\title{
SAÚDE PÚBLICA EM
}

\section{TEMPOS PANDEMICOS}

\section{VOLUME 1}

Organizador:

Raul Sousa Andreza

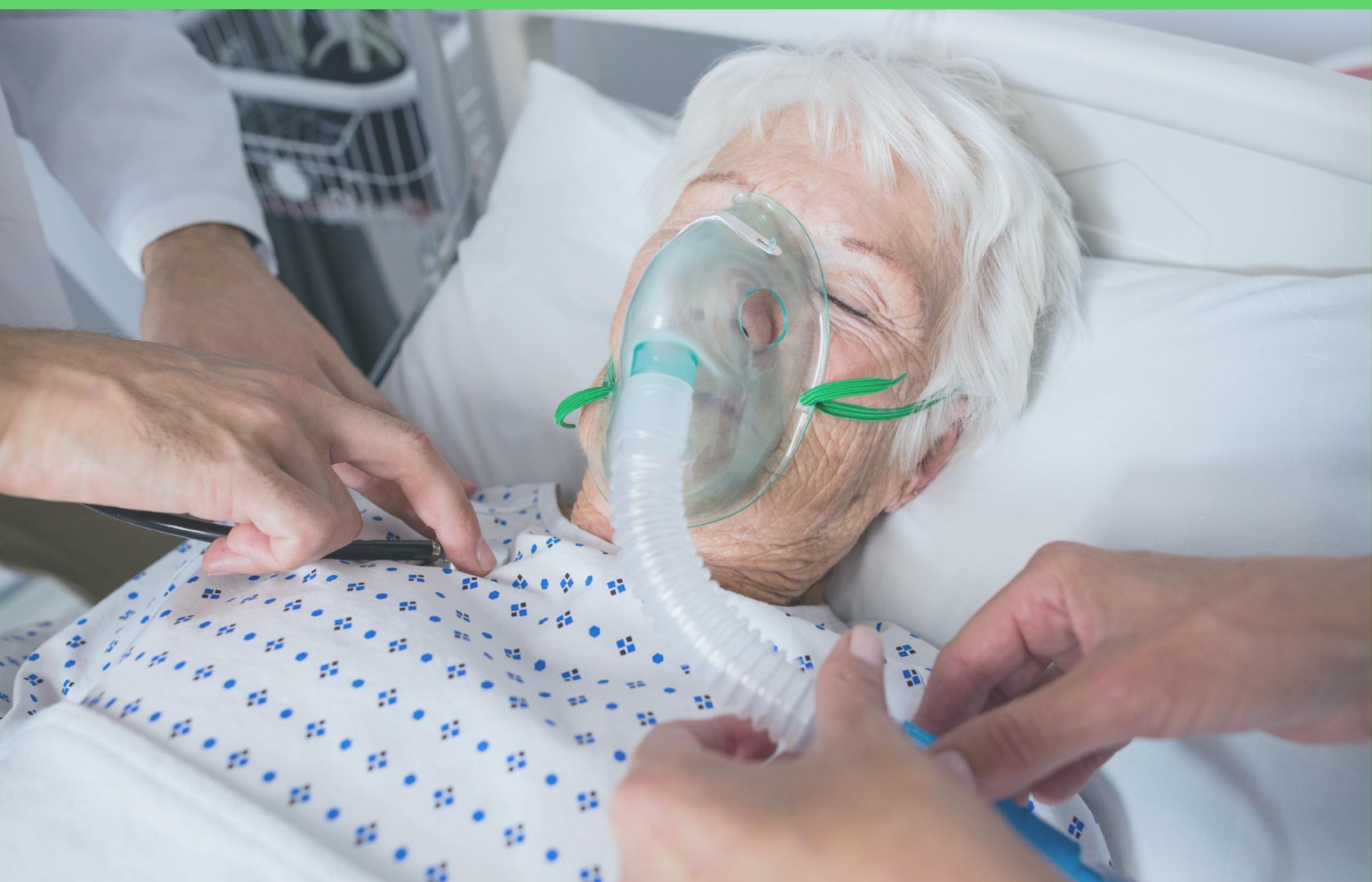


EDITORA

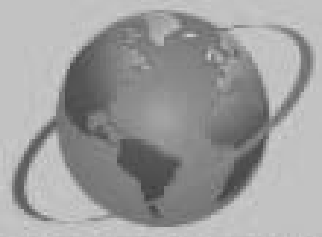

OMNIS SCIENTIA

\section{SAÚDEE PÚBUICA BM}

\section{THEMIPOS PANDENMICOS}

\section{VOLUME 1}

Organizador:

Raul Sousa Andreza

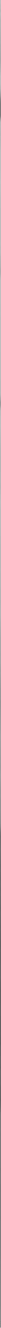


Editora Omnis Scientia

SAÚDE PÚBLICA EM TEMPOS PANDÊMICOS

Volume 1

$1^{a}$ Edição

TRIUNFO - PE

2021 


\section{Editor-Chefe}

Me. Daniel Luís Viana Cruz

Organizador (a)

Prof. Me. Raul Sousa Andreza

\section{Conselho Editorial}

Dra. Pauliana Valéria Machado Galvão

Dr. Wendel José Teles Pontes

Dr. Walter Santos Evangelista Júnior

Dr. Cássio Brancaleone

Dr. Plínio Pereira Gomes Júnior

Editores de Área - Ciências da Saúde

Dra. Camyla Rocha de Carvalho Guedine

Dra. Cristieli Sérgio de Menezes Oliveira

Dr. Leandro dos Santos

Dr. Hugo Barbosa do Nascimento

Dr. Marcio Luiz Lima Taga

Dra. Pauliana Valéria Machado Galvão

\section{Assistentes Editoriais}

Thialla Larangeira Amorim

Andrea Telino Gomes

Imagem de Capa

Freepik

\section{Edição de Arte}

Vileide Vitória Laranjeira Amorim

\section{Revisão}

Os autores

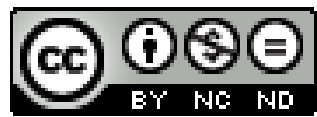

Este trabalho está licenciado com uma Licença Creative Commons - AtribuiçãoNãoComercial-SemDerivações 4.0 Internacional.

O conteúdo abordado nos artigos, seus dados em sua forma, correção e confiabilidade são de responsabilidade exclusiva dos autores. 
Dados Internacionais de Catalogação na Publicação (CIP) (eDOC BRASIL, Belo Horizonte/MG)

S255 Saúde pública em tempos pandêmicos [livro eletrônico] / Organizador Raul Sousa Andreza. - Triunfo, PE: Omnis Scientia, 2021.

286 p. : il.

Formato: PDF

Requisitos de sistema: Adobe Acrobat Reader

Modo de acesso: World Wide Web

Inclui bibliografia

ISBN 978-65-88958-41-4

DOI 10.47094/978-65-88958-41-4

1. Ciências da saúde. 2. Saúde pública. 3. Pandemia. I. Andreza, Raul Sousa.

CDD 610

Elaborado por Maurício Amormino Júnior - CRB6/2422

\author{
Editora Omnis Scientia \\ Triunfo - Pernambuco - Brasil \\ Telefone: +55 (87) 99656-3565 \\ editoraomnisscientia.com.br \\ contato@editoraomnisscientia.com.br
}




\section{PREFÁCIO}

A incidência do novo coronavírus no Brasil é preocupante. No entanto, a saúde pública do país e o sistema de atendimento visa abranger a diversidade que o Brasil apresenta, o sistema único de saúde (SUS) tem como base a integralidade, a universalidade e a equidade de todos os pacientes e trabalhadores. Instituído assim, para democratizar toda a saúde brasileira, tem o interesse de ofertar serviços de qualidade a população. Portanto, ao longo da história de sua consolidação a saúde pública foi deixado de lado e passou a gerar grandes problemas aos profissionais atuantes.

De fato, os estudos desenvolvidos no âmbito da saúde pública se propõem a articular conhecimentos de diferentes saberes e fazeres fornecendo subsídios teóricos, práticos e metodológicos que contribuem positivamente para a construção de estratégias e políticas públicas que visam o desenvolvimento de informações e ações em prol de uma saúde de qualidade para toda comunidade.

O presente livro é composto por 24 capítulos elaborados por autores pertencentes às ciências da saúde e suas áreas afins com o objetivo de proporcionar conhecimentos e compartilhar experiências e resultados de estudos desenvolvidos em várias localidades brasileiras e que visam à elucidação de diferentes situações de saúde.

Em nossos livros selecionamos um dos capítulos para premiação como forma de incentivo para os autores, e entre os excelentes trabalhos selecionados para compor este livro, o premiado foi o capítulo 02, intitulado "VACINAS CONTRA COVID-19: UMA BREVE DESCRIÇÃO POR MEIO DE REVISÃO INTEGRATIVA“. 


\section{SUMÁRIO}

CAPÍTULO 1

A IMPORTÂNCIA E OS DESAFIOS DA ESTRATÉGIA DE SAÚdE DA FAMÍLIA NO ENFRENTAMENTO DA COVID-19

Romana Erica Tavares Grangeiro Pinto

Wyara Ferreira Melo

Maria Amanda Laurentino Freires

Patrício Borges Maracajá

Aline Carla de Medeiros

José Cândido da Silva Nóbrega

Manoel Marques de Souto Nóbrega Filho

Túlio Alberto de Oliveira Sousa

Mônica Valéria Barros Pereira

Vicente Saraiva dos Santos Neto

Hozanna Estrela Celeste

Gabriela Rocha Pordeus dos Santos

DOI: 10.47094/978-65-88958-41-4/17-26

CAPÍTULO 2

VACINAS CONTRA COVID-19: UMA BREVE DESCRIÇÃO POR MEIO DE REVISÃO INTEGRATIVA

Fernanda Lima Marçal

Isabela Figueiredo e Souza

Maria Eduarda Coelho Gomes

Larissa Lima Torres

Isabela Campbell Santos

Thamara Lóren Lima

Ludmilla Vieira Magalhães

Maria Eduarda Sirina Pereira

Lucas Viana de Oliveira 
Larissa da Silva Torres França

Natan Fiorotti da Silva

Milena de Oliveira Simões

DOI: 10.47094/978-65-88958-41-4/27-41

CAPÍTULO 3

AÇÃO EM SAÚdE E A IMPORTÂNCIA DO USO CORRETO DAS MÁSCARAS PARA PREVENÇÃO DE INFECÇÕES RESPIRATÓRIAS

Soniely Nunes de Melo

Rafael Belarmino de Souza Lima

Tarcísio Correia Sposito

Rayana Ribeiro Trajano de Assis

Nayara Sandriele Santana de Souza

Bruna Rafaella Santos Torres

Flávio José Alencar de Melo

Davi Silva de Jesus

Carlos Henrique Bezerra de Siqueira

Izabelle Barbosa da Silva

Marcos André de Holanda Prudente Pessoa

Ana Marlusia Alves Bomfim

DOI: 10.47094/978-65-88958-41-4/42-51

CAPÍTULO 4

CONHECIMENTOS DAS EQUIPES MULTIPROFISSIONAIS DA ESTRATÉGIA DE SAÚDE DA FAMIILIA SOBRE COVID-19 E GESTAÇÃO

Priscilla dos Santos Nascimento

Michelle Araújo Moreira

DOI: 10.47094/978-65-88958-41-4/52-65 
CARTILHA EDUCATIVA SOBRE A COVID-19 PARA A PROTEÇÃO DA COMUNIDADE ACADÊMICA DO CAMPUS BELÉM DO IFPA

Lidineusa Machado Araujo

Maria de Nazaré Pereira Rodrigue Martins

Gabriela Priscila de Lima Carvalho

Fernanda Rafaela de Souza Rebelo da Costa

Michelle da Silva Pereira

Andréa de Melo Valente

Maria Helena Cunha Oliveira

Antônio Marcos Mota Miranda

DOI: 10.47094/978-65-88958-41-4/66-75

CAPÍTULO 6 .76

A SAÚdE DOS PROFISSIONAIS DE SAÚDE QUE ATUAM NA LINHA DE FRENTE NA PANDEMIA DO COVID - 19: REVISÃO INTEGRATIVA

João Lucas Ferreira Andrade

Léa Bianch Lima

Luana Kellen Nogueira Epitácio

Maria Eduarda Alves Vasconcelos

Antônio Augusto Ferreira Carioca

Carlos Antônio Bruno da Silva

Eudóxia Sousa de Alencar

DOI: 10.47094/978-65-88958-41-4/76-89

CAPÍTULO 7 .90

OS EFEITOS DA PANDEMIA NA COMPULSÃO ALIMENTAR: UMA REVISÃO DE LITERATURA

Lais Pontes de Miranda Cerqueira

Tarcio Goncalves sobral

Isadora Bianco Cardoso de Menezes

DOI: 10.47094/978-65-88958-41-4/90-97 
O IMPACTO DA PANDEMIA DO COVID - 19 SOBRE A SEGURANÇA ALIMENTAR E NUTRICIONAL: REVISÃO INTEGRATIVA

Bruna Sousa Barbosa

Igor Matheus Cruz de Oliveira

João Lucas Ferreira Andrade

Léa Bianch Lima

Luana Kellen Nogueira Epitácio

Maria Eduarda Alves Vasconcelos

Antônio Augusto Ferreira Carioca

Carlos Antônio Bruno da Silva

Eudóxia Sousa de Alencar

DOI: 10.47094/978-65-88958-41-4/98-104

CAPÍTULO 9 105

A UTILIZAÇÃO DOS PROTOCOLOS DE USO DO GUIA ALIMENTAR PARA A POPULAÇÃO BRASILEIRA: RELATO DE EXPERIÊNCIA

Bárbara Santos Amorim

Lis Chaves Marinho

Isadora Bianco Cardoso de Menezes

DOI: 10.47094/978-65-88958-41-4/105-114

CAPÍTULO 10 .

O IMPACTO DA COVID-19 EM PORTADORES DE SÍNDROME METABÓLICA

Laura Rasul de Lima

Ana Beatriz Amaral Vieira

Gabriella Neiva Reis

Ingrid Ravenna Liberalino Lima

DOI: 10.47094/978-65-88958-41-4/115-122 
SIMPLIFICANDO A HANSENÍASE: PROJETO DE INTERVENÇÃO REALIZADO EM

MEIO DIGITAL DURANTE A PANDEMIA

Amanda Almeida Lima

Ana Beatriz Sousa Santos

Francisco Vittor Miranda e Araújo

Jesamar Correia Matos Filho

João Ferreira de Paula Neto

Maria Clara de Freita Albano

Manoel Cícero Viana de Lima

Pedro Schmitt Martins Paiva Matos

Ruddy Mariano Maia Cysne Guerra

Samuel Carvalho Vasconcelos

Thaine Mirla Rocha

Elaine Lopes Bomfim

DOI: 10.47094/978-65-88958-41-4/123-131

CAPÍTULO 12.

DESCOMPLICANDO A HANSENÍASE: PROJETO DE INTERVENÇÃO REALIZADO EM UNIDADE DE SAÚDE NO PERÍODO DA PANDEMIA

Adália Stefanny de Araujo Cavalcante

Giovanna Giffoni Souza do Nascimento

Iêda de Freitas Martins Jota

Isabel Camila Araújo Barroso

Kaio Rangel Freitas Guimarães

Laís Mesquita de Sousa

Monique dos Santos Chaves

Manoel Victor Freires Vieira

Matheus Macedo Braga Coelho

Thaine Mirla Rocha

Elaine Lopes Bomfim 
CAPÍTULO 13. 140

HANSENÍASE: UM ESTUDO EPIDEMIOLÓGICO NA REGIÃO DO MÉDIO ARAGUAIAMATO GROSSO

Flavia Rodrigues Santana

Josilene Dália Alves

DOI: 10.47094/978-65-88958-41-4/140-150

CAPÍTULO 14 151

MUNICÍPIOS COM ALTA INCIDÊNCIA DE TUBERCULOSE EM MATO GROSSO: CONHECER PARA INTERVIR EM TEMPOS DE PANDEMIA

Larissa Machado Bellé

Yasmim Paloma Abreu Silva

Alessandro Rolim Scholze

Josilene Dália Alves

DOI: 10.47094/978-65-88958-41-4/151-162

CAPÍTULO 15 163

SAÚDE INTEGRAL DA MULHER EM CONTEXTO PANDÊMICO: REVISÃO INTEGRATIVA DE LITERATURA

Lohana Guimarães Souza

Tailande Venceslau Carneiro

Letícia Grazielle Santos

DOI: 10.47094/978-65-88958-41-4/163-175

CAPÍTULO 16 176

ASSISTÊNCIA DE ENFERMAGEM NO OUTUBRO ROSA E PERFIL EPIDEMIOLÓGICO DAS MULHERES: RELATO DE EXPERIÊNCIA

Helena Pereira de Souza

Laura Letícia Perdição Guerra

Luana Fernandes e Silva 
Thales Philipe Rodrigues da Silva

Alessandra Lage Faria

Helen Carine Ferreira Balena

Érica Moreira de Souza

Bruna Luíza Soares Pinheiro

Lorena Medeiros de Almeida Mateus

Flávia Duarte de Oliveira Ribeito

Bianca Maria Oliveira Luvisaro

Fernanda Penido Matozinhos

DOI: 10.47094/978-65-88958-41-4/176-188

CAPÍTULO 17 189

AGRAVAMENTO DAS DOENÇAS PSIQUIÁTRICAS DURANTE O PERÍODO DE ISOLAMENTO SOCIAL: UMA BREVE REVISÃO DE LITERATURA

Hellen Kristina Magalhães Brito

Gabriela Teixeira Lima

Ana Laura Fernandes Tosta

Laura Beatriz Caitano de Oliveira

Maria Paula Ricardo Silva

Mariana Vieira Garcia de Carvalho

Nathália Siriano Costa

Mayara Rita Figueredo

Mabel Fernandes Rocha

Helena Maria Mendes Marques

Kaio Murilo Santana Corrêa

Ana Flávia Buiatte Andrade

DOI: 10.47094/978-65-88958-41-4/189-200 
ATUAÇÃO DO PSICÓLOGO EM TEMPO DE PANDEMIA: UMA REVISÃO DE ESTUDOS NACIONAIS

Gabriel Rigamonte

Sueli Souza

Wilson Quiroz

Daniel Bartholomeu

Fernando Pessotto

Cintia Heloína Bueno

Fernanda Helena Viana Garcia

DOI: 10.47094/978-65-88958-41-4/201-213

CAPÍTULO 19

CRIAÇÃO DE UM APLICATIVO VOLTADO PARA UMA UNIDADE BÁSICA DE SAÚdE EM FORTALEZA

Isabella Araujo Duarte

Giovanna Rolim Pinheiro Lima

Idna Lara Goes de Sena

Laura Figueiredo Leite

Letícia Cavalcante Lócio

Livian Araújo Camelo Gomes

Maria Regina Cardoso Linhares Oliveira Lima

Maria Tereza Linhares Cardoso

Pedro Henrique Cardoso Nogueira

Rafael Albuquerque Franco

Rodrigo Carvalho Paiva

Berta Augusta Faraday Sousa Pinheiro

DOI: 10.47094/978-65-88958-41-4/214-229 
TELEMEDICINA E SUAS VARIÁVEIS NO CENÁRIO DE PANDEMIA MUNDIAL: UMA REVISÃO DE LITERATURA

Raniere Canteiro Garcia Lhamas

Andressa Marcolino Campos

Douglas Ferreira Lima

Gabriel Souza Ferreira Oliveira

Guilherme de Mendonça Lopes Beltrão

Luciana de Paula Santana

Nícollas Nunes Rabelo

DOI: 10.47094/978-65-88958-41-4/230-237

CAPÍTULO 21 238

MONITORIA ACADÊMICA NO ENSINO REMOTO: PERSPECTIVAS E DESAFIOS EM TEMPOS PANDÊMICOS

Felipe Gabriel Assunção Cruz

Givanildo Bezerra de Oliveira

Marcílio Delan Baliza Fernandes

Ana Lúcia Moreno Amor

DOI: 10.47094/978-65-88958-41-4/238-248

CAPÍTULO 22

A DOENÇA DO OLHO SECO NA SÍINDROME DE SJÖGREN

Bruna Rafaella Santos Torres

Carlos Eduardo Ximenes da Cunha

Carlos Henrique Bezerra de Siqueira

Flavia Emanuelly Alves França Gomes

Santília Tavares Ribeiro de Castro e Silva

Anna Caroline Guimarães Gomes

Laís Rytholz Castro

Dennis Cavalcanti Ribeiro Filho 
Lara Medeiros Pirauá de Brito

Marina Viegas Rezende Ribeiro

DOI: 10.47094/978-65-88958-41-4/249-259

CAPÍTULO 23 260

FEIRAAGROECOLÓGICA: DIFICULDADES E POTENCIALIDADES DE UM CIRCUITO CURTO DE COMERCIALIZAÇÃO

Maria Rita Garcia de Medeiros

Rônisson Thomas de Oliveira Silva

Maria Natalícia de Lima

Ana Beatriz Macêdo Venâncio dos Santos

DOI: 10.47094/978-65-88958-41-4/260-269

CAPÍTULO 24 270

AVALIAÇÃO DO IMPACTO DE UMA REDE SOCIAL EM USUÁRIOS DE UMA UNIDADE BÁSICA DE SAÚDE EM FORTALEZA

Luiz Gerson Gonçalves Neto

Letícia Cavalcante Lócio

Carlos Alexandre Leite Pereira Filho

Henrique Sousa Costa

Maria Helena dos Santos Macedo

Lígia Bringel Olinda Alencar

Berta Augusta Faraday Sousa Pinheiro

Isaac Dantas Sales Pimentel

DOI: 10.47094/978-65-88958-41-4/270-280 


\section{CAPÍTULO 4}

\section{CONHECIMENTOS DAS EQUIPES MULTIPROFISSIONAIS DA ESTRATÉGIA DE SAÚDE DA FAMÍLIA SOBRE COVID-19 E GESTAÇÃO}

\section{Priscilla dos Santos Nascimento ${ }^{1}$;}

Universidade Estadual de Santa Cruz (UESC), Ilhéus/BA.

http://lattes.cnpq.br/7008758440665266

https://orcid.org/0000-0002-2429-2357

\section{Michelle Araújo Moreira²}

Universidade Estadual de Santa Cruz (UESC), Ilhéus/BA.

http://lattes.cnpq.br/7310566538375094

https://orcid.org/0000-0002-6998-8665

RESUMO: O cenário referente à associação da doença Coronavírus Disease-2019 (COVID-19) com a gestação permanece marcado pela escassez de evidências científicas. Assim, apresenta-se como objetivo geral: Analisar os conhecimentos das equipes multiprofissionais da Estratégia Saúde da Família sobre COVID-19 e gestação. E como objetivos específicos: Levantar os conhecimentos das equipes multiprofissionais da ESF sobre COVID-19 e gestação; discutir os conhecimentos das equipes multiprofissionais da ESF sobre COVID-19 e gestação à luz da Organização Pan-Americana de Saúde (OPAS) e da Organização Mundial de Saúde (OMS). Trata-se de um estudo qualitativo, descritivo e exploratório. O cenário do estudo foi composto pela Unidade de Saúde da Família (USF) Nossa Senhora da Vitória, USF Nelson Costa I e II e USF Ilhéus II, pertencentes ao município de Ilhéus-Bahia. Os sujeitos foram membros das equipes multiprofissionais, conforme critérios de inclusão. Os dados foram coletados remotamente, através de plataformas digitais como "Google Meet" ou "Zoom". O instrumento de coleta de dados foi um roteiro de entrevista semiestruturada. A análise de conteúdo temática dos dados foi respaldada por Bardin. Constatou-se ambiguidade nos resultados, analisados como (des)conhecimento, apresentando amplo entendimento quanto aos aspectos gerais da doença, porém com lacunas e divergências referentes à associação da COVID-19 com a gestação; e (des)alinhamento com as recomendações apresentadas pela OPAS e OMS quanto ao manejo e orientações sobre prevenção. Conclui-se que equipes multiprofissionais da ESF devem estar capacitadas e atualizadas para acompanhar as gestantes através de uma assistência em saúde integral e resolutiva.

PALAVRAS-CHAVE: Coronavírus. Estratégia Saúde da Família. Gravidez. 
ABSTRACT: The scenario regarding the association of Coronavirus Disease-2019 (COVID-19) with pregnancy remains marked by the scarcity of scientific evidence. Thus, it presents itself as a general objective: To analyze the knowledge of the multidisciplinary teams of the Family Health Strategy on COVID-19 and pregnancy. And as specific objectives: To raise the knowledge of the ESF multiprofessional teams on COVID-19 and pregnancy; discuss the knowledge of the ESF multiprofessional teams on COVID-19 and pregnancy in the light of the Pan American Health Organization (PAHO) and the World Health Organization (WHO). This is a qualitative, descriptive and exploratory study. The study scenario was composed by the Family Health Unit (USF) Nossa Senhora da Vitória, USF Nelson Costa I and II and USF Ilhéus II, belonging to the municipality of Ilhéus-Bahia. The subjects were members of the multiprofessional teams, according to the inclusion criteria. The data were collected remotely, through digital platforms such as "Google Meet" or "Zoom". The data collection instrument was a semi-structured interview script. Thematic content analysis of the data was supported by Bardin. There was ambiguity in the results, analyzed as (un) knowledge, presenting a broad understanding of the general aspects of the disease, but with gaps and divergences regarding the association of COVID-19 with pregnancy; and (dis) alignment with the recommendations presented by PAHO and WHO regarding management and prevention guidelines. It is concluded that multidisciplinary teams of the FHS must be trained and updated to accompany the pregnant women through comprehensive and resolutive health care.

KEY-WORDS: Coronavirus. Family Health Strategy. Pregnancy.

\section{INTRODUÇÃO}

A disseminação da doença Coronavírus Disease-2019, abreviada pela Organização Mundial de Saúde (OMS) por COVID-19 como referência ao ano de descoberta da doença e seu agente etiológico, teve início com a suspeita de uma doença infecciosa que rapidamente ultrapassou as barreiras geográficas, caracterizando-se como Emergência de Saúde Pública de Importância Internacional, evoluindo para um quadro de pandemia em 2020. Assim, surgiram os primeiros indícios de infecção por uma nova cepa de coronavírus humano, o SARS-CoV-2 que, até então, ainda não havia sido identificada. Desde a sua descoberta, o número de infectados já ultrapassa 140 milhões, quando somados todos os continentes, com mais de 3 milhões de óbitos (OPAS, 2020a; HUI et al., 2020).

A infecção por SARS-CoV-2 apresenta um alto índice de transmissibilidade pessoa a pessoa, principalmente, por meio de gotículas respiratórias disseminadas por tosse ou espirro, ou contato direto com um indivíduo infectado, sintomático ou não. Apesar de esta ser a forma de transmissão sustentada por evidências, ainda não foram descartadas outras vias, como por exemplo, transmissão vertical, seja durante a gestação, parto ou amamentação, ou por meio de relação sexual através de secreção vaginal ou sêmen (BRASIL, 2020a). 
O espectro clínico é amplo, variando desde sintomas respiratórios leves a quadros de pneumonia e Síndrome Respiratória Aguda Grave (SRAG), ainda sem medicamento específico indicado para tratamento definitivo. As medidas de suporte adotadas até o momento visam controlar os sintomas, prevenir a transmissão comunitária e, principalmente, evitar as possíveis complicações que são observadas com maior índice nos indivíduos mais vulneráveis, entre eles idosos, indivíduos com doenças crônicas e respiratórias, gestantes em qualquer idade gestacional, puérperas, além dos imunodeprimidos que compõem o grupo com condições clínicas de risco para o desenvolvimento de complicações devido a infecção pelo novo coronavírus (BRASIL, 2020b; 2020c).

Para além do contágio e possibilidade de complicações, ainda persistem dúvidas na comunidade científica quanto a questões específicas relacionadas a COVID-19, incluindo sua associação com a gestação. Até o momento, os trabalhos publicados relacionando estes dois fatores consistem basicamente em relatos de casos, recomendações de especialistas ou diretrizes de sociedades licenciadas (AMORIM, 2020).

Em contrapartida, o panorama brasileiro se caracteriza com um quadro de transmissão sustentada da COVID-19, atingindo diretamente o Sistema Único de Saúde (SUS) em todas as suas redes de atenção, dentre elas a atenção básica, que tem como referência as unidades com programa Estratégia Saúde da Família (ESF), entendido como uma estratégia de reorganização do modelo assistencial. Integrado à rede, este programa consolida-se como porta de entrada preferencial para o sistema de saúde do país, atendendo a comunidade local ao ofertar promoção e prevenção à saúde de maneira integral (DOS SANTOS CRUZ et al., 2017).

Uma vez que a ESF desenvolve, entre outras, ações voltadas à saúde da mulher, portanto também presta assistência às gestantes e que as equipes multiprofissionais em saúde atuantes nestas unidades devem estar capacitadas e atualizadas quanto aos agravos e protocolos adequados para o manejo e resolução destes, esta pesquisa justifica-se pela necessidade de investigar os conhecimentos dos profissionais de saúde da ESF sobre COVID-19 e o período gestacional.

A partir do exposto, surge então a seguinte questão norteadora: Quais os conhecimentos das equipes multiprofissionais da ESF sobre COVID-19 e gestação? A fim de responder tal questão, definiu-se como objetivo geral: Analisar os conhecimentos das equipes multiprofissionais da ESF sobre COVID-19 e gestação. E como objetivos específicos: Levantar os conhecimentos das equipes multiprofissionais da ESF sobre COVID-19 e gestação; discutir os conhecimentos das equipes multiprofissionais da ESF sobre COVID-19 e gestação à luz da Organização Pan-Americana de Saúde (OPAS) e da Organização Mundial de Saúde (OMS).

\section{MATERIAL E MÉTODOS}

Trata-se de um estudo qualitativo, descritivo e exploratório, definida quanto à natureza por pesquisa básica e quanto ao procedimento desenvolveu-se a partir de pesquisa de campo. Sabe-se que a pesquisa qualitativa, enquanto método científico, abarca os fenômenos que atingem os sujeitos em seus contextos sociais compreendendo os fenômenos como passíveis de análise quanto à sua 
intensidade para, assim, direcionar a compreensão da realidade. Constitui-se como descritivo, pois essa modalidade metodológica, em geral, descreve e analisa características de uma população ou de um fenômeno, podendo também estabelecer possíveis relações entre variáveis. Define-se por caráter exploratório, pois trabalha a possibilidade de aprofundar-se no problema investigado, trazendo a possibilidade da construção de hipóteses de investigação (TAQUETTE, 2016; GIL, 2017; FILHO; FILHO, 2015).

O cenário da pesquisa foi composto pela Unidade de Saúde da Família (USF) Nossa Senhora da Vitória, USF Ilhéus II e USF Nelson Costa I e II, pertencentes ao município de Ilhéus. A cidade de Ilhéus, localizada ao sul do Estado da Bahia, apresenta uma área territorial de $1.588,555 \mathrm{~km}^{2} \mathrm{e}$ aproximadamente 162.327 habitantes (IBGE, 2019).

Realizou-se investigação com 25 participantes, membros que compõem as equipes multiprofissionais das USF mencionadas, selecionados a partir da sua categoria profissional, sendo: seis enfermeiras, uma médica, quatro técnica(o)s de enfermagem e 14 agentes comunitários de saúde. Estes atenderam aos seguintes critérios de inclusão: ter vínculo empregatício com a sua referida unidade de saúde por um período superior a seis meses. Os critérios de exclusão empregados foram: profissionais em processo de adoecimento físico ou mental com afastamento das atividades laborais.

Considerando as recomendações dos órgãos de saúde quanto ao isolamento e distanciamento social por causa da pandemia da COVID-19, a coleta de dados fora realizada remotamente, via plataformas virtuais como "Google Meet" e "Zoom". Estas plataformas possibilitam a realização de videochamadas em tempo real com recursos para gravação/registro das mesmas, viabilizando a transcrição e análise dos dados coletados. As gravações serão arquivadas por cinco anos e excluídas após esse período.

Foi utilizado como instrumento de coleta de dados um roteiro de entrevista semiestruturada, no qual consta investigação quanto ao perfil sociodemográfico do(a)s participantes e perguntas abertas relacionadas ao tema. Para garantir privacidade e sigilo, todo(a)s foram nomeado(a)s por codinomes que foram escolhidos pelo(a)s próprio(a)s depoentes antes das entrevistas.

A análise dos dados foi respaldada por Bardin, constituindo a análise de conteúdo temática. Esta técnica demonstra-se eficaz em pesquisas que utilizam aplicação de questões abertas ou as que realizam entrevistas, possibilitando interpretar os significados das respostas e as intenções, atingindo-se assim, o conteúdo em si. Possibilita a análise a partir de três etapas: pré-análise, na qual se sistematizam as ideias iniciais; seguida pela exploração do material, com a análise propriamente dita; e finaliza-se pelo tratamento das respostas, as inferências e as interpretações dos resultados (BARDIN, 2016).

Ressalta-se que o projeto foi submetido ao Comitê de Ética em Pesquisa (CEP) da Universidade Estadual de Santa Cruz (UESC) para avaliação, considerando as questões éticas abordadas nas Resoluções no 466/2012 e nº 510/2016, aprovado sob parecer nº 3.990.524 (BRASIL, 2012; 2016). 
A pesquisa com os profissionais de saúde atuantes nas USF Nossa Senhora da Vitória, Ilhéus II e Nelson Costa I e II, localizadas no município de Ilhéus, somente teve início após análise e aprovação final do CEP, seguindo os critérios de inclusão e exclusão estabelecidos previamente, mediante esclarecimento prévio, claro e de fácil entendimento da natureza, objetivos, métodos, benefícios, riscos e/ou desconfortos, discutidos com os sujeitos a partir da leitura do Termo de Consentimento Livre e Esclarecido (TCLE) durante a vídeochamada.

O processo de consentimento livre e esclarecido dos profissionais de saúde para participação na pesquisa consistiu em um procedimento virtual, no qual o(a)s depoentes foram orientados a consentir verbalmente com a sua participação no estudo, cientes da gravação deste aceite.

\section{RESULTADOS E DISCUSSÃO}

Quanto ao perfil sociodemográfico do(a)s participantes, constatou-se que a faixa etária está compreendida entre 29 e 60 anos de idade. No que se refere ao gênero foram entrevistados três homens e 22 mulheres. No que diz respeito ao tempo de formação, os profissionais indicaram atuar como enfermeiras, técnico(a)s de enfermagem, agentes comunitários de saúde e médica com tempo de formação variável entre cinco e 25 anos.

Posterior à identificação do perfil, iniciou-se a leitura atentiva das entrevistas, análise do conteúdo e a categorização dos dados, destacadas a seguir:

(Des)conhecimento das equipes multiprofissionais sobre Covid-19 e os aspectos do ciclo gravídico

Neste estudo o entendimento das equipes multiprofissionais sobre o novo coronavírus está relacionado, principalmente, a aspectos como as repercussões clínicas da doença no sistema respiratório, a alta capacidade de transmissibilidade, a variabilidade do espectro clínico e a suscetibilidade para complicações em indivíduos que apresentam comorbidades, como pode-se observar nos seguintes depoimentos:

[...] é um vírus letal que ataca o sistema respiratório [...] é altamente contagioso, com maior letalidade para pessoas com comorbidades [...] (Carlos/Técnico de Enfermagem).

O novo coronavirus é uma infecção viral de RNA [...] e que tem muitas cepas [...] tem variabilidade muito grande de sintomatologia [...] (Mariana/Médica).

Estes resultados demonstram conformidade do conhecimento das equipes com as evidências científicas considerando que a infecção por SARS-CoV-2 apresenta, fundamentalmente, complicações respiratórias como pneumonia, síndrome da angústia respiratória aguda, insuficiência respiratória e outros quadros clínicos graves, com maior probabilidade de infectar indivíduos com comorbidades crônicas, a exemplo das doenças cardiovasculares e diabetes (BRASIL, 2020b; CHEN et al., 2020).

Quanto à sintomatologia relacionada ao novo coronavírus, o(a)s entrevistado(a)s demonstraram amplo conhecimento baseado nos relatos dos pacientes atendidos nas USF ou na experiência da atividade laboral conforme apontam as falas a seguir: 
[...] tosse, coriza, nariz congestionado, dor de garganta, febre, diarreia, irritação nos olhos, perda de paladar e olfato, dores no corpo, moleza, desânimo (Lucas/Agente Comunitário de Saúde).

[...] muito variáveis [...] tosse, sensação de febre [...], dispneia muito frequente, diarreia [...] dor no peito, tontura, fraqueza, mas o mais comum é dor de cabeça e dor no corpo [...]

(Mariana/Médica).

As manifestações clínicas da COVID-19 apresentam, entre os sintomas mais comuns, febre, tosse seca, dispneia, dor torácica, fadiga e mialgia. Outros sintomas são relatados na literatura, ainda que sejam apresentados por um número menos expressivo de infectados, destacando-se cefaleia, dor abdominal, tontura, diarreia, náuseas e vômitos (HARAPAN et al., 2020).

Pontuados os aspectos gerais acerca da infecção, seguiu-se a investigação a respeito da associação da COVID-19 com a gestação. Quando questionados sobre complicações no curso da gravidez provocadas pela doença em questão, uma parcela expressiva dos sujeitos não descarta a possibilidade de complicações, com frequência justificando e enfatizando nas falas a crença na fragilidade do corpo gravídico o que, segundo os relatos, aumentaria os riscos de complicações em gestantes:

[...] pode trazer complicações [...] no início da gravidez pode causar o aborto, porque pode diminuir a questão do transporte de oxigênio da placenta para o bebê [...] ou algumas outras sequelas que a gente ainda não tem conhecimento (Vanessa/Agente Comunitária de Saúde).

[...] a gravidez já traz algumas doenças como, por exemplo, diabetes gestacional ou hipertensão, e se você associa isso ao vírus, com certeza vai trazer alguma consequência (Maria/Técnica de Enfermagem).

[...] pode trazer complicações. Pelo que eu vi em alguns relatos, casos de descolamento de placenta e outros que resultaram até em óbitos materno e fetal (Alice/Enfermeira).

Os estudos que eu li ainda não demonstravam uma manifestação própria do coronavírus para a gravidez [...] só orientavam que houvesse maior cuidado por conta da dificuldade do manejo [...](Mariana/Médica).

Notou-se que o conhecimento demonstrado pelo(a)s entrevistado(a)s diverge das evidências científicas, pois ainda que as mudanças fisiológicas decorrentes da gestação levem a uma predisposição a infecções, inclusive respiratórias, estudos apontam que a evolução da COVID-19 não parece ser pior nas gestantes, assemelhando-se ao quadro clínico apresentado em adultos não gestantes, quando infectados. No entanto, é sensata a preocupação em relação à infecção pelo SARS-CoV-2 nesta população baseado nas complicações que, quando surgem, apresentam-se como graves ou críticas, conforme observado também em outras infecções respiratórias no ciclo gravídico-puerperal, a exemplo de outros coronavírus (SARS e MERS) e o vírus influenza H1N1 (BRASIL, 2020d). 
Quanto à suscetibilidade das gestantes ao contágio e a inclusão destas no grupo de risco do novo vírus, o(a)s participantes demonstram ambiguidade nas informações em relação à literatura evidenciando um desconhecimento, por parte de alguns profissionais, quanto ao enquadramento de mulheres grávidas na categoria supracitada, conforme pode-se observar:

Não estão no grupo de risco, mas apresentam maior risco de complicações (Mariana/ Médica).

[...] tem que ter um cuidado maior [...] mas não quer dizer que a gestante esteja em uma situação de risco, como risco de pegar coronavírus ou morrer porque está grávida, essas coisas não (Pedro/Agente Comunitário de Saúde).

[...] a gestante, pra mim, é sim do grupo de risco [...] é vulnerável, porque ela está carregando outro ser junto com ela [...](Bruna/Agente Comunitária de Saúde).

[...] a gestante se enquadra no risco de qualquer paciente adulto que a gente atende(Ricardo/ Enfermeiro).

Sabe-se que, inicialmente, as gestantes não estavam inclusas no grupo com condições clínicas de risco para o desenvolvimento de complicações se infectadas pelo novo coronavírus. No entanto, posteriormente foram adicionadas à categoria visto que, determinadas mudanças no sistema imunológico provocados pela própria gravidez podem afetar a resposta imune de gestantes. Entre as alterações que podem ocorrer, destaca-se a tolerância imunológica desenvolvida pelo corpo materno para prevenir rejeição ao feto, fator esse que deve ser considerado em uma possível infecção por COVID-19 (BRASIL, 2020c; BARREIRA et al., 2015).

Quanto à transmissão vertical durante a gestação, ao aumento no risco de aborto e em relação a anomalias no feto possivelmente provocadas pelo SARS-CoV-2, percebeu-se expressivo desconhecimento e incerteza pelas equipes justificadas, muitas vezes, pela escassez ou inexistência de contato com gestantes infectadas e por não terem sido orientados quanto a isso através da educação permanente do serviço:

[...] a partir do momento que não acontece o transporte adequado de oxigênio através da placenta para o bebê e pode acontecer o aborto, talvez seja por essa questão de transmitir intrauterino mesmo. Não tenho conhecimento adequado, mas acredito que sim [...] (Vanessa/ Agente Comunitária de Saúde).

[...] li em algumas situações que parece que a transmissão foi sim materno-fetal, agora não sei se foi imediatamente após o parto ou se foi transmissão transplacentária [...] existem recémnascidos com coronavírus [...] então a transmissão, provavelmente, é materno-fetal(Mariana/ Médica).

[...] acredito que podem ocorrer complicações na gestação e no parto, mas sobre a transmissibilidade eu não sei responder ao certo(Marta/Técnica de Enfermagem). 
[...] só ouvi falar do abortamento e do parto prematuro nos casos mais complicados, mas anomalia não $[\ldots]($ Larissa/Enfermeira).

[...] não sei informar [...] não fui orientada sobre isso [...] (Gabriela/Agente Comunitária de Saúde).

Pesquisas recentes sugerem que a transmissão vertical intrauterina no terceiro trimestre da gestação é improvável. Quanto ao primeiro e o segundo trimestre, as investigações ainda estão em andamento. Ademais, amostras de líquido amniótico, placenta, sangue do cordão umbilical, leite materno e secreção vaginal não evidenciaram presença do vírus. Também não há evidências suficientes para sugerir aumento no risco de aborto associado à infecção, nem para indicar que o vírus SARSCoV-2 seja teratogênico, nem resulte em dismorfologias fetais ou anomalias congênitas, assim como não foi observado em infecções por SARS e MERS. Em todas as situações mencionadas reconhecese a necessidade de mais análises futuras (YANG e LIU, 2020; SCHWARTZ e GRAHAM, 2020; RCOG, 2020).

\section{Estratégias adotadas pelas equipes multiprofissionais da ESF sobre COVID-19 e gestação versus} as recomendações da OPAS e OMS

A ESF configura-se como protagonista para a efetivação do pré-natal na Atenção Primária à Saúde (APS) do SUS, cenário no qual as equipes multiprofissionais destas unidades acompanham mulheres no período gestacional e no puerpério, de acordo com suas atribuições. Neste nível de atenção desenvolvem-se, entre outras, ações voltadas ao cuidado e à atenção integral à saúde da mulher que significam, na prática, assistência capaz de garantir que o acompanhamento pré-natal atenda questões voltadas à prevenção de doenças, ao diagnóstico e tratamento de agravos que podem afetar o curso da gestação (GUIMARÃES et al., 2018; SEHNEM et al., 2020).

Nesse sentido, as condutas adotadas pelas equipes multiprofissionais nas USF devem ser baseadas no que é preconizado ou indicado pelos órgãos e instituições de saúde reconhecidas, seguindo critérios e evidências científicas que tragam segurança e resultados satisfatórios a todos os envolvidos na assistência, direta ou indiretamente. Esta pesquisa buscou, para além de aspectos clínicos e epidemiológicos, esclarecer junto aos entrevistado (a)s quais as orientações tem sido indicadas às gestantes, pretendendo assim analisá-las a partir das recomendações que são preconizadas pela OPAS e OMS.

Sobre as recomendações voltadas às gestantes em relação à prevenção do contágio do novo coronavírus, entre os resultados destacam-se orientações que, em geral, não diferem das medidas sinalizadas aos demais indivíduos predispostos à infecção, exemplificados a seguir: 
[...] orientações que são gerais, mantendo o isolamento, tomando os devidos cuidados de higienização: lavagem de mãos, uso de máscara, uso de álcool em gel, evitar contato com outras pessoas [...](Marta/Técnica de Enfermagem).

[...] que usem máscara e que estejam sempre lavando as mãos [...] evitar muito contato, lavar sempre as mãos em um local que tenha água e sabão, ou então levar o álcool gel, usar máscara, trocar a máscara a cada 2 horas [...] são cuidados que todos nós temos que ter e elas também [...] (Talita/Enfermeira).

A prevenção que a gente passaparaas gestantes é a mesma que a gente passa pra todo mundo [...] os cuidados são os mesmos: se manter afastada de aglomeração, estar sempre fazendo higienização das mãos, usar máscara sempre [...](Laís/Agente Comunitária de Saúde).

Neste aspecto, os depoimentos analisados alinham-se com as orientações da OPAS e da OMS quanto à prevenção dos seres humanos para a infecção por SARS-CoV-2, visto que enfatizam o uso de máscaras e a higienização das mãos para todos os indivíduos, sendo eles gestantes ou não. As máscaras devem ser usadas como parte importante de um conjunto de medidas que visam controlar a transmissão do coronavírus, ainda que se reconheça que, quando não associadas a outras estratégias de prevenção, são insuficientes para fornecer um nível adequado de proteção contra a COVID-19 (OPAS, 2020a).

As orientações das equipes multiprofissionais e as recomendações de precaução para a prevenção e controle de infecções (PCI) dos órgãos de saúde mencionados anteriormente incluem, além das máscaras, a higienização frequente das mãos, o distanciamento físico quando possível, atenção à etiqueta respiratória, limpeza e desinfecção do ambiente, controle de temperatura e evitar locais com aglomeração de pessoas (OPAS, 2020b; OMS, 2020a).

Investigou-se também a respeito do fluxo de atendimento das unidades às quais os profissionais estão vinculados, quanto ao manejo da assistência em saúde e as medidas que devem ser adotadas em caso de suspeita e confirmação de coronavírus em uma gestante. No que se referem a essas medidas os resultados revelam divergências quanto à compreensão de como, quando e quais medidas devem ser adotadas, evidenciando insegurança e acesso limitado às informações sobre a rede municipal de atendimento para casos de coronavírus em mulheres grávidas:

Eu sinceramente não sei. O que eu faço é só notificar para a enfermeira. O que vem depois eu não sei(Samuel/Agente Comunitário de Saúde).

A gestante precisa ser imediatamente isolada e acompanhada pela vigilância epidemiológica. Não é nem o posto de saúde que tem que acompanhar ela, é a vigilância epidemiológica que tem que prestar toda assistência de enfermeiro, médico, tudo isso(Luana/Técnica de Enfermagem).

Notificação, encaminhamento para o serviço médico, monitoramento dos sinais vitais [...] (Sara/Enfermeira). 
[...] no manejo inicial essa paciente é incluída no nosso monitoramento, sendo reavaliada a cada $24 \mathrm{~h}$ ou $48 \mathrm{~h}[\ldots .$.$] e nesse período de reavaliação a gente vê se apresentou algum novo$ sintoma ou se tem algum que está persistindo [...] se for o caso, a gente já encaminha para outro serviço para avaliação da equipe de obstetrícia, para ver se tem alguma coisa que possa estar afetando a gestação [...](Mariana/Médica).

A partir das evidências científicas disponibilizadas e divulgadas pela OMS, o Brasil e os demais países, conforme sua capacidade e contexto, desenvolveram um conjunto de medidas sistematizadas em protocolos de manejo/fluxo de atendimento como resposta da rede de atenção em saúde à COVID-19. Esses protocolos visam reduzir a transmissão e a mortalidade associada ao novo coronavírus e, portanto, são válidos em território nacional, complementando ou alterando a rotina de determinados serviços de saúde em vários níveis de atenção tendo impacto também na atenção primária (OMS, 2020a).

A partir dessas medidas, definiu-se que o manejo clínico na APS tem como referência a ESF que deve assumir papel resolutivo frente aos casos leves e de identificação precoce, assim como encaminhamento rápido e correto dos casos graves para serviços de referência. Deve-se priorizar o atendimento para os indivíduos que compõem o grupo de risco, incluindo gestantes em qualquer idade gestacional, em um fluxo de atendimento que envolve a identificação de casos suspeitos de COVID-19 e adoção de medidas para evitar contágio nas unidades e de estratificação da gravidade do quadro clínico (BRASIL, 2020b).

Apesar de mencionarem com frequência os serviços de referência para os quais as gestantes devem ser encaminhadas, não há uniformidade no discurso das equipes sobre como se dá esse encaminhamento ou o atendimento nas próprias unidades em que atuam. Observa-se também que, enquanto equipes multiprofissionais em saúde, há pouco alinhamento das informações referentes ao atendimento quando estas excedem suas próprias atribuições profissionais e são, muitas vezes, desconhecidas ou ignoradas. A notificação dos casos é pouco ou raramente mencionada.

Quanto à rotina do serviço como, por exemplo, a assistência pré-natal, as(os) participantes relataram mudanças no fluxo de atendimento, alterações no espaço físico, assim como adaptações no acompanhamento/monitoramento das famílias, como o contato por Whatsapp, respeitando ao máximo as orientações quanto ao distanciamento social:

A demanda é agendada mais ou menos por horário, reduzimos a quantidade de pessoas por turno para que elas venham e não fiquem aglomeradas. Pedimos que evitem acompanhantes (Sara/Enfermeira).

Foi reduzido. Como os outros programas saíram, sobrou mais espaço pra que não haja uma aglomeração das gestantes. Mas o pré-natal continua sendo feito, não tem como ser interrompido(Maria/Técnica de Enfermagem). 
[...] nas nossas visitas de rotina, mesmo porque não podemos adentrar nas casas, a gente fica na parte de fora e mantém uma certa distância, mas o bom é que as gestantes continuam fazendo acompanhamento [...] (Mateus/Agente Comunitário de Saúde).

Funcionando agendamento por horário [...] uma entra e a outra fica esperando lá fora, porque o espaço lá é mais arejado. E estamos evitando atender acima de cinco usuárias. Então elas entram, fazem o atendimento e quando termina a enfermeira faz a higienização da sala. Só então é que se chama a outra gestante. Nem estão ficando na sala de espera justamente para evitar aglomeração (Bárbara/Agente Comunitária de Saúde).

Assim como as equipes multiprofissionais entrevistadas tem atuado, as agências de saúde enfatizam a importância do isolamento social e a adoção de formas de comunicação à distância, a reorganização dos serviços e estratégias para a continuidade do cuidado como o espaçamento de tempo entre os atendimentos. Orienta-se que as consultas de pré-natal devem seguir as rotinas habituais dentro do possível, considerando o risco gestacional e a situação singular de cada mulher, sendo priorizadas as consultas durante o terceiro trimestre para as gestantes em condição de baixo risco e para todas as mulheres com gestação de alto risco. Durante as consultas recomenda-se investigar a presença de sintomas gripais e/ou contato recente com pessoas infectadas pela COVID-19 (FEBRASGO, 2020; OMS, 2020b).

A partir do exposto, percebe-se que os resultados evidenciam, de maneira geral, ambiguidade nos depoimentos dos sujeitos, revelando divergências e lacunas no conhecimento no que diz respeito à associação da COVID-19 com a gestação. Observou-se um (des)conhecimento das equipes multiprofissionais investigadas, pois algumas informações estão bem definidas e embasadas cientificamente, enquanto outras estão sustentadas pelo senso comum e carecem de maior esclarecimento ou até mesmo do contato dos sujeitos com situações que exijam melhor preparo para a assistência que deve ser prestada. Tratando-se das estratégias utilizadas pelas equipes constatouse, como na categoria anterior, (des)alinhamento com as recomendações apresentadas pela OPAS e OMS, ainda que em menores níveis, em especial quanto ao manejo clínico das gestantes.

\section{CONCLUSÃO}

A Estratégia de Saúde da Família destaca-se na atenção integral à saúde das mulheres, entre as quais incluem-se as gestantes e, portanto, faz-se necessário que as equipes multiprofissionais deste nível de atenção atuem na identificação, manejo e resolução dos agravos que podem vir a acometêlas, a exemplo da COVID-19.

Assim, a pesquisa com as equipes multiprofissionais da ESF revela uma ambiguidade nos resultados, que pode ser entendido como um (des)conhecimento referente às informações disponíveis na literatura sobre COVID-19 e gestação; e (des)alinhamento das estratégias adotadas relacionadas às recomendações preconizadas pela OPAS e OMS. Ainda que as informações gerais a respeito do novo coronavírus estejam respaldadas por evidências científicas, como conceito, medidas de prevenção 
e sintomatologia, quando questionados sobre a relação da COVID-19 com a gestação, há muitas lacunas e/ou informações que tem como base o senso comum em especial sobre o comportamento do vírus no corpo gravídico, suas implicações para o binômio mãe-bebê, assim como determinadas divergências quanto às orientações dos órgãos de saúde mencionados anteriormente.

As limitações encontradas no decorrer deste estudo referem-se a escassez de evidências científicas conclusivas, considerando a simultaneidade do desenvolvimento da pesquisa com as análises que estão sendo realizadas sobre o tema. Admite-se a necessidade de novas investigações, posteriormente, quando se espera que as lacunas do conhecimento existentes atualmente quanto à associação da COVID-19 com a gestação, tenham sido esclarecidas.

\section{DECLARAÇÃO DE INTERESSES}

Nós, autoras deste artigo, declaramos que não possuímos conflitos de interesses de ordem financeira, comercial, político, acadêmico e pessoal.

\section{REFERÊNCIAS}

AMORIM, Melânia Maria Ramos de. Protocolo de atendimento de pacientes com COVID-19 (infecção suspeita ou confirmada). Instituto de Saúde Elpídio de Almeida (ISEA), Campina Grande, 44 p. 2020. Disponível em: https://www.dropbox.com/s/m9xxmwkbstpa6zl/PROTOCOLO $\% 20$ DE\%20CRISE\%20COVID19\%20ISEA\%20\%20vers\%C3\%A3o\%2003.04.2020.pdf?dl=0 2020. Acesso em: 09 abr. 2020.

BARDIN, Laurence. Análise de Conteúdo. São Paulo: Edições 70, 2016.

BARREIRA, Joana Filipa et al. Alterações imunológicas e da função tiroideia na gravidez e no período pós-parto. ArqMed, v. 29, n. 2, p. 56-60, abr. 2015. Disponível em: http://www.scielo.mec.pt/ scielo.php?script=sci_abstract\&amp;pid=S087134132015000200004\&amp;lng=pt\&amp;nrm=iso. Acesso em: 03 out. 2020.

BRASIL. Ministério da Saúde. Conselho Nacional de Saúde. Resolução no 466, de 12 de dezembro de 2012. Trata sobre as diretrizes e normas regulamentadoras de pesquisa envolvendo seres humanos. Diário Oficial da União, Brasília(DF).

BRASIL. Ministério da Saúde. Conselho Nacional de Saúde. Resolução nº 510, de 7 de abril de 2016. Trata sobre as diretrizes e normas regulamentadoras de pesquisa em ciências humanas e sociais. Diário Oficial da União, Brasília (DF).

BRASIL. Ministério da Saúde. Secretaria de Vigilância em Saúde. Guia de Vigilância Epidemiológica - Emergência de Saúde Pública de Importância Nacional pela Doença pelo Coronavírus 2019. Brasília (DF): MS, 2020a.

BRASIL. Ministério da Saúde. Secretaria de Atenção Primária à Saúde. Departamento de Ações 
Programáticas Estratégicas. Protocolo de Manejo Clínico do Coronavírus (COVID-19) na Atenção Primária à Saúde. 5. ed. Brasília (DF): MS, 2020 b.

BRASIL. Ministério da Saúde. Secretaria de Atenção Especializada à Saúde. Departamento de Atenção Hospitalar, Domiciliar e de Urgência. Protocolo de manejo clínico da Covid-19 na Atenção Especializada. 1. ed. Brasília (DF): MS, 2020c.

BRASIL. Ministério da Saúde. Secretaria de Atenção Primária à Saúde. Departamento de Ações Programáticas Estratégicas. NOTA TÉCNICA No 12/2020-COSMU/CGCIVI/DAPES/SAPS/MS. Brasília (DF): MS, 2020d.

CHEN, Nanshan et al. "Epidemiologicalandclinicalcharacteristicsof 99 cases of 2019 novel coronavirus pneumonia in Wuhan, China: a descriptivestudy”. The Lancet, [s.1.], v. 395, n. 10223, p. 507-13, fev. 2020.

DOS SANTOS CRUZ, Joanderson et al. Avaliação do acesso à Estratégia Saúde da Família na perspectiva dos usuários no município de Santo Antônio de Jesus-Bahia, Brasil. Ver SaludPública, Bogotá, 2017, v. 19, n. 5, p. 641-48. Disponível em: http:/www.scielo.org.co/scielo. php?script=sci_arttext\&pid=S0124-00642017000500641\&lng=pt\&nrm=iso. Acesso em: 23 nov. 2020.

GIL, Antônio Carlos. Como elaborar projetos de pesquisa. 6. ed. São Paulo: Atlas, 2017.

GUIMARAES, Wilderi Sidney Gonçalves et al. Acesso e qualidade da atenção pré-natal na Estratégia Saúde da Família: infraestrutura, cuidado e gestão. Cad Saúde Pública, Rio de Janeiro, v.34, n.5, e00110417, 2018. Disponível em: http:/www.scielo.br/scielo.php?script=sci_ arttext\&pid=S0102-311X2018000505001\&lng=en\&nrm=iso. Acesso em: 24 nov. 2020.

FEDERAÇÃO BRASILEIRA DAS ASSOCIAÇÕES DE GINECOLOGIA E OBSTETRÍCIA (FEBRASGO). Recomendações FEBRASGO para o GO em tempos de COVID-19. 2020. Disponível em: https://www.febrasgo.org.br/es/covid19/item/975-recomendacoes-febrasgo-para-o-go-emtempos-de-covid-19. Acesso em 02 nov. 2020.

FILHO, Milton Cordeiro Farias; FILHO, Emilio Arruda. Planejamento da pesquisa científica. 2. ed. São Paulo: Atlas, 2015.

HARAPAN, Harapan et al. "Coronavirusdisease 2019 (COVID-19): A literaturereview”. Journalofinfectionandpublichealth, v. 13, n. 5, p. 667-73, mai. 2020.

HUI, David S et al. The continuing 2019-nCoV epidemicthreatof novel coronavirusesto global health The latest 2019 novel coronavirusoutbreak in Wuhan, China. InternationalJournalOfInfectiousDiseases, [s.1.], v. 91, p. 264-66, fev. 2020.

INSTITUTO BRASILEIRO DE GEOGRAFIA E ESTATÍSTICA (IBGE). IBGE cidades: Bahia/ Ilhéus. 2019. Disponível em: <https://cidades.ibge.gov.br/brasil/ba/ilheus/panorama>. Acesso em: 31 mar. 2020. 
ORGANIZAÇÃO MUNDIAL DE SAÚDE (OMS). Actualización de La estrategia frente a la covid-19. 2020a. Disponível em: https://www.who.int/docs/default-source/coronaviruse/covidstrategy-update-14april2020_es.pdf?sfvrsn=86c0929d_10. Acesso em 24 nov. 2020.

ORGANIZAÇÃO MUNDIAL DE SAÚDE (OMS). Mantenimiento de losservicios de saludesenciales: orientaciones operativas enel contexto de la COVID-19. Orientacionesprovisionales. 2020b. Disponível em: https://apps.who.int/iris/bitstream/handle/10665/334360/WHO-2019-nCoVessential_health_services-2020.2-spa.pdf. Acesso em: 24 nov. 2020.

ORGANIZAÇÃO PANAMERICANA DE SAÚdE (OPAS). Folha informativa - COVID-19 (doença causada pelo novo coronavírus). 2020a. Disponível em: $<$ https://www.paho.org/bra/index. php?option=com_content\&view=article\&id=6101:covid19\&Itemid=87>. Acesso em: 12 out. 2020 .

ORGANIZAÇÃO PANAMERICANA DE SAÚDE (OPAS). Transmissão do SARS-CoV-2: implicações para as precauções de prevenção de infecção. Resumo científico. 2020b. Disponível em: https://iris.paho.org/bitstream/handle/10665.2/52472/OPASWBRACOVID-1920089_por. pdf?sequence=1\&isAllowed=y. Acesso em: 02 out. 2020

PRODANOV, Cleber Cristiano; FREITAS, Ernani Cesar. Metodologia do Trabalho Científico: Métodos e Técnicas de pesquisa e do Trabalho Acadêmico. 2. ed. Novo Hamburgo (RS): Feevale, 2013.

ROYAL COLLEGE OF OBSTETRICIANS AND GYNAECOLOGISTS (RCOG). Coronavirus (COVID-19) Infection in Pregnancy: information for healthcareprofessionals. Information for healthcareprofessionals. 2020. Disponível em: https://www.rcog.org.uk/globalassets/documents/ guidelines/2020-10-14-coronavirus-covid-19-infection-in-pregnancy-v12.pdf. Acesso em: 03 nov. 2020 .

SCHWARTZ, DavidA; GRAHAM,Ashley L. Potential Maternal andInfantOutcomesfromCoronavirus 2019-nCoV (SARS-CoV-2) InfectingPregnantWomen: lessonsfromsars, mers, andotherhumancoronavirusinfections. Viruses, [s.1.], v. 12, n. 2, p. 194-210, fev. 2020.

SEHNEM, Graciela Dutra et al. Consulta de pré-natal na atenção primária à saúde: fragilidades e potencialidades da intervenção de enfermeiros brasileiros. Ver Enf Ref. Coimbra, v. ser V, n. 1, p. e19050-e190050, jan. 2020. Disponível em: http://www.scielo.mec.pt/scielo.php?script=sci_ arttext\&pid=S087402832020000100005\&lng=pt\&nrm=iso. Acesso em: 24 nov. 2020.

TAQUETTE, Stella Regina. Análise de dados de pesquisa qualitativa em saúde. In: Congresso IberoAmericano em Investigação Qualitativa, 5., 2016, Porto. Atas. Porto: CIAIQ, 2016. v. 2, p. 524-33.

YANG, Ziyi; LIU, Yi. Vertical Transmission of Severe Acute Respiratory Syndrome Coronavirus 2: A Systematic Review. Am J Perinatol, v. 37, n. 10, p. 1055-70, ago. 2020. 


\section{Índice Remissivo}

$\mathbf{A}$

Acesso aos psicólogos 201, 203, 204

Acesso às redes sociais 43

Agricultor e o consumidor 260

Agricultores familiares 260, 263, 266

Alterações do metabolismo 115

Ambiente virtual 215

Ansiedade 77, 78, 81, 82, 83, 84, 86, 87, 91, 92, 94, 95, 117, 121, 190, 192, 193, 194, 197, 204, 207, 208, 209

Aplicativo 136, 215, 217, 218, 219, 220, 221, 222, 223, 224, 225, 226, 227, 228, 243, 271, 272, 273, 274, 278, 280

Aprendizagem 239

Assistência social 163, 171, 205

Associação da covid-19 com a gestação 52

Astrazeneca (universidade de oxford) 28, 30

Atenção primária 25, 124

Atenção primária à saúde (aps) 19, 59, 107, 125, 177, 178

Atendimento ambulatorial 105

Atendimento on-line 201, 203

Atendimento remoto 202, 205

Atividades econômicas 28

Autoanticorpos 250

Autoridades sanitárias 67, 69, 73, 278

C

Capacidade de defesa do organismo 115

Características dos imunizantes 28, 30

Cartilha educativa sobre a covid-19 67, 69, 70

Ceratoconjuntivite sicca $(\mathrm{kcs}) 250$

Combate à hanseníase 133

Comercialização 38, 260, 261, 262, 263, 264, 265, 266, 267, 268, 269

Compulsão alimentar 90, 92, 93, 94, 95, 96

Consumo de alimentos 105

Contaminação 43, 44, 45, 47, 49, 72, 73, 74, 78, 117, 135, 179, 191, 193, 195, 197

Coronavac (sinovac) 28, 29

Cuidados individuais e coletivos $67,69,73$

Cuidados preventivos ao covid-19 115

D

Depressão 78, 81, 82, 83, 87, 91, 92, 94, 95, 110, 172, 190, 192, 193, 194, 197, 207, 208, 209

Desemprego 21, 99, 103, 153

Desenvolvimento do câncer 177, 185

Desigualdade em saúde 163

Desinformação 29, 208 
Diabetes 21, 56, 57, 95, 105, 106, 107, 108, 114, 116, 117, 118, 119, 120, 181, 183, 186

Direito humano à alimentação adequada 103, 260

Disseminação da informação 67,73

Distanciamento social 25, 48, 55, 61, 68, 73, 90, 95, 126, 130, 192, 195, 202, 279

Distúrbios psicológicos 190

Doença infecciosa 53, 125, 133, 134, 140, 141, 151, 152

Doença multifatorial 250

Doenças 21, 24, 28, 29, 43, 54, 56, 57, 59, 69, 91, 95, 106, 107, 108, 115, 116, 117, 119, 120, 129, 133, 137, 140, 142, $149,161,163,164,167,171,172,173,177,185,186,193,194,195,207,210,217,222,223,227,250,271$, $272,273,278,279$

Doenças crônicas 105, 171, 218, 221, 227, 273

Doenças negligenciadas 140, 142

\section{$\mathbf{E}$}

Educação em saúde 19, 24, 43, 46, 48, 50, 67, 69, 73, 105, 108, 133, 134, 135, 136, 137, 145, 179, 185, 215, 216, 217, $227,228,238,240,248,271,272,274$

Efeitos colaterais $28,30,31,32,33$

Enfermagem 50, 56, 57, 58, 60, 61, 88, 89, 122, 149, 161, 176, 177, 178, 180, 188, 227, 240, 280

Enfermidade epidêmica 238

Enfrentamento da covid-19 18, 19, 20, 23, 24, 25

Epidemiologia 114, 140, 148, 149, 160, 161, 242, 248

Equipamentos de proteção 24, 43, 47, 78, 171, 197

Equipes multiprofissionais $52,54,55,56,59,60,61,62$

Estratégia saúde da família (esf) 18, 19, 23, 54

Estratégias nutricionais 105

Estresse 77, 78, 81, 82, 83, 86, 87, 88, 89, 94, 172, 190, 192, 193, 197, 206, 207, 209, 210, 211

Etiologia autoimune 250

Evidências científicas 29, 52, 56, 57, 59, 61, 62, 63, 76, 79, 99, 101, 190

F

Feiras agroecológicas 260, 261, 264

Ferramenta tecnológica de saúde 215

Fortalecimento da saúde púbica 67

Frequência cardíaca 230

G

Gestação 52, 53, 54, 57, 58, 59, 61, 62, 63, 224

Glândulas exócrinas 250, 251, 252, 253

Guia alimentar 105, 107, 108, 112, 113

$\mathbf{H}$

Hábitos alimentares 90, 92, 94, 95, 111, 112

Hanseníase 124, 125, 126, 127, 128, 130, 131, 133, 134, 135, 136, 137, 138, 139, 140, 141, 142, 143, 144, 145, 146, $147,148,149$

Herança genética 115

Hipertensão 57, 95, 105, 107, 108, 114, 115, 116, 118, 119, 120, 121, 181, 183, 186, 228, 229, 274, 276,278 
Hiposecreção lacrimal 250

I

Idosos 19, 21, 23, 54, 105, 108, 134, 137

Impacto da pandemia 99

Imunização 28, 29, 30, 35, 38, 179, 183, 186

Inclusão 20, 22, 52, 55, 56, 58, 75, 77, 79, 101, 163, 165, 167, 215, 226, 268

Infecções 28, 29, 38, 43, 44, 48, 57, 59, 60, 78, 165, 172, 180, 227, 251

Infecções respiratórias virais $43,44,48$

Informação de qualidade 271, 279

Informação em saúde 67,69

Informação sobre a hanseníase 124

Instabilidade econômica 99, 103

Instrumento de prevenção 43

Intervenção psicológica 202

Isolamento $23,48,55,60,62,81,90,91,92,94,100,142,164,177,179,190,191,192,193,194,195,196,197,198$, $199,203,204,205,207,208,210,242,261$

Isolamento social 23, 26, 62, 90, 92, 94, 100, 142, 164, 177, 179, 190, 191, 192, 194, 195, 196, 197, 198, 203, 205, 207, 208, 210, 242

$\mathbf{J}$

Janssen (johnson \& johnson) 28, 29, 30

$\mathbf{L}$

Lesões cutâneas 140, 143

Linha de frente 76, 78, 79, 81, 84, 86, 87, 88, 171, 195, 197, 202, 206, 207, 208, 210

M

Má qualidade do sono 77,87

Máscaras faciais 43

Material digital 43, 46

Medição biométrica 230

Medidas de isolamento 26, 28, 194

Medidores de glicose 230

Metodologia pico (problema, intervenção, contexto, resultado) 76, 79, 99, 101

Monitores do centro de ciências da saúde (ccs) 238

Monitoria 238, 239, 240, 241, 242, 243, 244, 246

Morbidade 115, 119

Mortalidade materna 163, 171, 172

Mudanças nos estilos de vida 90, 92, 93

Mycobacterium leprae 135, 140, 141, 146

N

Níveis socioeconômicos 43, 46

Novas modalidades de interações 238

Novo coronavírus sars-cov-2 18, 19 
Olho seco 250, 251, 252, 253, 254, 255, 256, 257, 258

Organização mundial de saúde (oms) 19, 52, 53, 54, 68, 92, 191

Organização pan-americana de saúde (opas) 52, 54

Órgãos oficiais de saúde 238, 247

Outubro rosa $177,178,179,184,185$

$\mathbf{P}$

Padrões dietéticos 115

Pandemia 18, 19, 20, 21, 23, 24, 25, 26, 28, 31, 38, 45, 47, 48, 53, 55, 68, 69, 73, 75, 76, 78, 79, 81, 82, 84, 86, 87, 88, $89,90,91,92,93,94,95,96,99,100,101,102,103,104,117,118,119,120,124,126,130,131,133,135,136$, $137,142,152,153,160,161,162,163,164,165,168,169,170,171,172,173,174,177,179,180,186,187$, 190, 191, 192, 193, 194, 195, 196, 197, 198, 199, 200, 203, 204, 205, 206, 207, 208, 210, 211, 212, 213, 238, $239,240,241,242,244,247,248,271,272,279,280$

Perfil alimentar 105

Perfil clínico 160, 177, 183, 185

Pfizer (pfizer e biontech) 28, 30

Políticas públicas de saúde 18, 24, 173, 204

Portadores de síndrome metabólica 115

Prática oftalmológica 250

Práticas do autocuidado 178,186

Pressão arterial 115,230

Prevenção 29, 33, 72, 170, 187, 221

Primeiros socorros 215, 218, 219, 225, 227, 273

Princípio ativo 28, 30, 33

Produção de alimentos 260

Profissionais de psicologia 202

Profissionais de saúde 18, 22, 24, 54, 56, 76, 78, 79, 81, 82, 84, 85, 86, 87, 89, 107, 125, 138, 151, 160, 170, 185, 194, $195,197,198,202,206,207,208,238$

Programa nacional de alimentação escolar (pnae) 99, 265

Promoção em saúde 43, 45, 271, 278

Propagação do vírus $43,47,48,67,69$

Q

Qualidade de vida 22, 47, 82, 106, 112, 155, 217, 226, 244, 250, 251, 254, 272, 278

Quarentena 90, 100, 121, 126, 170, 172, 190, 191, 192, 193, 194, 195, 199, 202, 206

$\mathbf{R}$

Recurso tecnológico 230

Redes sociais 43, 45, 46, 49, 133, 137, 194, 271, 272, 274, 278, 279

Responsabilidade individual e coletiva 18, 24

Restrição social 90, 92, 93 
Sars-cov- $20,21,29,30,31,32,33,34,35,38,39,40,45,49,53,56,57,58,59,60,65,88,120,163,164,165,168$, $173,191,199,238,239,240,242,243,247,248,279$

Saúde da família 18, 19, 20, 22, 23, 24, 25, 26, 52, 54, 55, 62, 64, 125, 135, 179, 187

Saúde da mulher 163, 166, 173

Saúde de maneira remota 133

Saúde dos profissionais $76,79,81,86,89$

Saúde dos trabalhadores $77,86,87$

Saúde integral 52, 163, 165, 167, 171

Saúde mental 78, 83, 88, 90, 93, 94, 95, 96, 172, 179, 190, 191, 192, 193, 194, 195, 196, 197, 198, 202, 203, 204, 206, $207,208,210,212,222,227,279$

Saúde psicológica 77,87

Saúde pública $6,20,28,38,69,73,82,120,125,134,139,141,148,149,160,168,169,170,171,172,177,179,197$, $199,208,211,238,240,242,247$

Secura ocular 250, 251, 257

Sedentarismo 94, 107, 115, 179

Segurança alimentar e nutricional 99, 100, 101, 102, 103, 104

Segurança alimentar nutricional (san) 99

Serviço de saúde 71, 115, 120, 180

Síndrome de sjögren (ss) 250, 251

Síndrome metabólica 115, 116, 117, 118, 119, 120

Sistema imunológico 58, 115, 117, 120

Sistema nacional de agravos de notificação (sinan) 140, 143

Situação de vulnerabilidade 90, 94, 95, 103

Superfície ocular 250, 254, 258

T

Tecnologia 66, 67, 69, 70, 137, 211, 231, 244

Tecnologias de informação e comunicação 208, 230

Teleatendimento 202, 204

Telemedicina 133, 170, 230, 232

Telessaúde 230, 236

Tempos pandêmicos 271

Terapêutica multidisciplinar 190

Teste de schirmer 250, 255

Testes oftalmológicos 250

Transmissão de informações 271, 279

Transtorno de ansiedade generalizada 190, 192

Tuberculose (tb) 151,152

$\mathbf{U}$

Unidade de saúde da família (usf) 52, 55

Uso das máscaras 43, 45, 48 
Vacina 28, 31, 32, 33, 34, 37, 39, 40, 70, 72, 159, 161, 183, 184, 186, 221

Variantes $28,30,31,34,35,38,49,78$

Vídeos educativos 215, 226

Violência contra a mulher $163,169,170,173,174$

$\mathbf{X}$

Xeroftalmia 250,251 


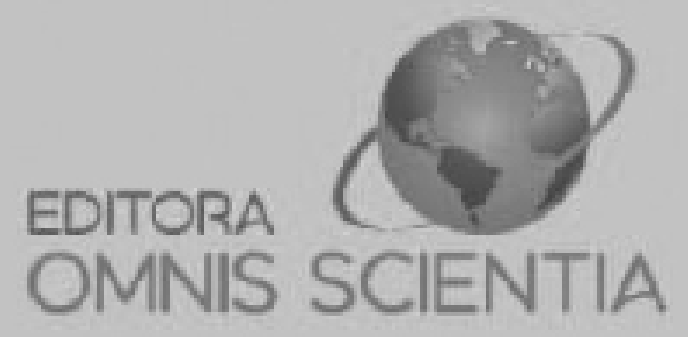

editoraomnisscientia@gmail.com M https://editoraomnisscientia.com.br/ @editora_omnis_scientia (0) https://wwww.facebook.com/omnis.scientia.9 $\boldsymbol{\oplus}$ +55 (87) 9656-3565 (C) 


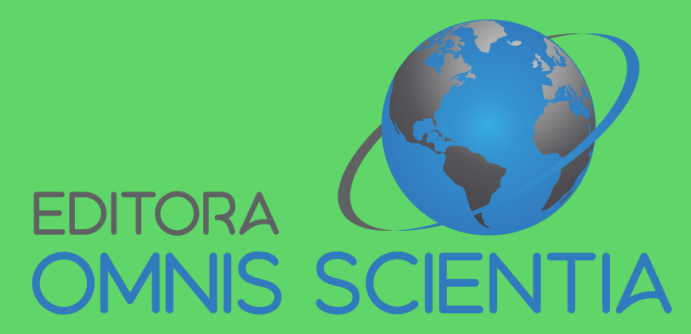

editoraomnisscientia@gmail.com M https://editoraomnisscientia.com.br/ (-) @editora_omnis_scientia (0) https://www.facebook.com/omnis.scientia.9 $\subsetneq$ +55 (87) 9656-3565 @ 